\title{
Implications biologiques de l'optimisation des irradiations
}

\author{
Raymond LATARJET, membre de l'Institut*
}

\section{RÉSUMÉ}

\begin{abstract}
Pour avoir participé à un groupe de travail réuni au Vatican par l'Académie pontificale des sciences, du 2 au 5 mai 1983, l'auteur soumet quelques réflexions personnelles que cette réunion lui a inspirées.
\end{abstract}

\section{ABSTRACT}

As a member of a working group set up by the Pontificia academia scientiarum, between May 2 nd and 5 th, 1983, the author confides some personal considerations over the main issues discussed at this meeting.

Le Saint-Siège s'intéresse très sérieusement à de nombreux problèmes scientifiques d'actualité et de caractère mondial. II y est contraint car il doit souvent prendre position sur tel ou tel d'entre eux. Selon le style habituel de l'Eglise, cette prise de position n'est jamais précipitée. Elle est précédée d'une étude approfondie dont la responsabilité est confiée à l'Académie pontificale des sciences. Celle-ci, en général, désigne un Groupe international restreint de spécialistes, qui, à la suite de ses travaux, répond par un texte à la question qui lui a été posée.

Dans le domaine qui intéresse Radioprotection, un tel groupe, dont j'eus I'honneur de faire partie, fut mis au travail en octobre 1981, et établit une "déclaration sur les conséquences de l'emploi des armes nucléaires". Le texte fut remis à S.S. Jean-Paul II. Celui-ci, après l'avoir adapté à son style personnel, et signé, chargea les membres du Groupe de le remettre en mains propres de sa part aux principaux chefs d'Etats respectifs (le Docteur Nicolas Bochkov à Leonid Breshnev, H. Hiatt à Ronald Reagan, Louis Leprince Ringuet à François Mitterrand, etc.) C'est dire combien sérieux sont ces travaux, et à quel niveau leurs conclusions sont exprimées.

De même, un peu plus tard, en septembre 1982, un autre texte fut mis au point : "Déclaration sur la prévention de la guerre nucléaire".

Tout récemment un groupe d'étude s'est réuni pendant quatre jours à l'Académie pontificale des sciences pour rédiger un texte susceptible de définir une prise de position du Saint-Siège sur le sujet qui figure en tête de cet article. $\mathrm{Ce}$ Groupe était placé sous la présidence du Pr. Carlos Chagas (Brésil), Président de l'Académie pontificale des sciences. II comprenait :

* Institut Curie (Biologie), 26, rue d'Ulm, 75005 Paris. 
Président : Carlos Chagas (Brésil), président de l'Académie pontificale des sciences.

MM. Gilbert Beebe (USA), ancien directeur du laboratoire d'Hiroshima.

Dan J. Beninson (Argentine), vice-président de la CIPR.

Merril Eisenbud (USA), director of the Laboratory for environment studies, Tuxedo, New York.

Mme Lidia Failla (Italie).

MM. W. Jacobi (Allemagne), directeur du Max Planck Institut für Strahlen und Umweltforschung, Neuherberg.

Raymond Latarjet (France).

Jérôme Lejeune (France), directeur de l'Institut de Progénèse, Paris.

Bo Lindell (Suède), président de la CIPR.

Carlo Polvani (Italie).

Giovanni Silini (Italie), secrétaire de l'UNSCEAR, Vienne (1).

Fritz H. Sobels (Hollande), directeur du Département de RadioGénétique, Leyde.

David Sowby (UK), secrétaire de la CIPR.

Le texte issu des délibérations a été remis au Pape, et demeure actuellement confidentiel. Mais les débats, à la fois vifs et courtois, qui furent échangés pendant quatre jours, m'ont inspiré un certain nombre de réflexions personnelles qui me semblent pouvoir intéresser les lecteurs de Radioprotection.

Le Groupe avait à étudier les facteurs économiques et sociaux de Poptimisation en prenant en considération à la fois l'individu et la population, ce qui soulève souvent des dilemmes. II devait placer le problème des radiations dans le contexte plus général de la pollution génotoxique, en particulier par les produits chimiques de ce type associés à la production d'énergie par combustion.

Dans ces discussions, qui restèrent toujours objectives et franches, on a vu s'opposer (comme il arrive immanquablement dans les discussions sur ce sujet) les optimistes et les pessimistes. L'optimiste dit: "Tant que vous n'aurez pas démontré qu'il y a un risque, je considérerai qu'il n'y en a pas". Le pessimiste dit : “Tant que vous n'aurez pas démontré qu'il n'y a pas de risque, je considérerai qu'il y en a un". Cette seconde attitude est évidemment sans issue lorsqu'on considére les risques à très long terme. C'est pourquoi elle est toujours adoptée par ceux qui désirent qu'il n'y ait pas d'issue.

\section{LES RÉPARATIONS ET LE SEUIL}

On dit souvent qu'en annulant ou compensant les effets des faibles doses, les phénomènes de réparation introduisent un seuil là où il n'y en avait pas. Tous les effets génotoxiques, quels qu'ils soient, commencent par des lésions du matériel génétique, notamment au niveau des acides nucléiques. Presque toutes les cellules sont dotées de remarquables mécanismes, constitutifs ou inductibles, qui assurent une certaine réparation des lésions au niveau de ces acides nucléiques, sauf chez les indi-

(1) UNSCEAR : Comité scientifique des Nations Unies pour l'étude des effets des rayonnements ionisants. 
vidus tarés par un défaut des systèmes de réparation (on connaît bien certaines maladies ainsi caractérisées). En fait, la situation est complexe.

Il faut d'abord distinguer les effets cellulaires où la réparation résulte de mécanismes biochimiques qui s'exercent au niveau de la macromolé cule d'ADN, et les effets tissulaires où la réparation résulte d'une repopulation par des cellules vivantes des cellules tuées.

En ce qui concerne ces derniers, la repopulation se produit tant que les tissus ou organes repopulateurs conservent une fraction intacte suffisante. Considérons, par exemple, le cas de la mœlle osseuse, l'un des tissus les plus radiosensibles. L'aplasie médullaire, qui pour être importante exige déjà de fortes doses (plusieurs centaines de rems), est suivie d'une intense multiplication des organes hémopoïétiques, rate, ganglions, mœlle osseuse elle-même. Lorsque ces organes n'ont pas été stérilisés, cette multiplication contribue assez vite à la repopulation et à la restitutio ad integrum. La preuve en est bien donnée par l'effet salvateur d'une injection de cellules médullaires isogéniques lorsque l'aplasie est trop importante et générale pour que l'organisme lui-même assure la repopulation. II est clair que dans ces effets tissulaires, ce type de restauration introduit un seuil souvent très étendu.

II en est autrement des effets cellulaires. Considérons, par exemple, la mort cellulaire et la production de mutations. Ce sont des phénomènes stochastiques. La courbe dose-effet est linéaire en coordonnées semilogarithmiques. La restauration de la lésion macromoléculaire est ellemême un phénomène stochastique. Chaque cellule qui a subi un coup létal (ou mutagène) a une certaine próbabilité de voir la conséquence de ce coup annulée par la réparation de la lésion moléculaire produite. La combinaison de deux phénomènes stochastiques aboutit à un phénomène stochastique, la réparation diminuant simplement la probabilité qu'un coup efficace ait eu lieu. La courbe dose-effet finale reste rectiligne, mais sa pente est moindre. La réparation équivaut à une réduction de la dose, dont on exprime numériquement le degré par "le facteur de réduction de dose", mais elle n'introduit pas un seuil. II arrive, parfois, que la réparation soit si efficace que la pente de la courbe dose-effet devient très faible, et commence par une portion si peu inclinée qu'elle équivaut à un véritable plateau. Et puis, la dose augmentant, le système de la réparation est à son tour endommagé. La réparation devient moins efficace, la pente s'accentue, et finit par être parallèle à la pente qui correspond à l'absence de réparation. La courbe théorique qui eut été rectiligne devient une courbe sigmoïde, sans seuil véritable, mais avec ce que l'on peut considérer comme un seuil pratique.

Enfin, il existe un cas très particulier, très important d'ailleurs, celui des mutations provoquées par une erreur de la réparation (réparation mutagène SOS). Cette fois-ci, la réparation non seulement n'introduit pas un seuil, mais introduit le phénomène qui augmente d'abord quand on augmente la dose et persiste tant que le système de réparation n'a pas été endommagé. C'est pourquoi d'ailleurs de nombreuses courbes doseeffet, pour l'induction de mutations comme pour la transformation cellulaire maligne in vitro, commencent par une partie ascendante rectiligne, pour atteindre un maximum, et diminuer ensuite. 


\section{R. LATARJET}

L'ensemble du Groupe a exprimé son accord sur l'ensemble de ces considérations, et en a profité pour mettre l'accent sur l'importance de la recherche fondamentale en radiobiologie qui est loin d'avoir encore répondu à tout ce que l'on attend d'elle en radioprotection.

\section{LA RADIOCANCÉRISATION, PHÉNOMĖNE NON STOCHASTIQUE}

En établissant ses recommandations la CIPR admet que la radiocancérisation est un phénomène stochastique, c'est-à-dire en particulier, qu'aux faibles doses la fréquence des cancers produits est proportionnelle à la dose. Ce concept très simple permet l'extrapolation des résultats expérimentaux acquis à forts débits de dose et à fortes doses jusqu'aux résultats inobservables qui seraient produits par les faibles doses, à faibles débits. C'est ainsi qu'on est arrivé à la notion qu'il faut 10000 hommes-rems pour produire un cancer. Cette proposition très simple est largement utilisée et rend des services évidents, mais elle est très probablement fausse.

Certes, le processus de radiocancérisation commence par une radiolésion, sans doute unique, dans le matériel génétique d'une cellule somatique. C'est une mutation somatique. Comme toutes les mutations, elle constitue un phénomène stochastique.

Mais la transformation maligne d'une cellule somatique n'est pas un cancer. Le cancer c'est la tumeur maligne, c'est-à-dire l'immense colonie de cellules nées de la première cellule transformée; pour que celle-ci donne sa colonie, il faut que cette colonie se développe. Or la plupart des organismes mammifères sont puissamment dotés pour s'opposer à ce développement clonal. II existe des réactions immunologiques, et sans doute d'autres, qui forment barrage ; et ceci est heureux, car la transformation maligne, tant spontanée que provoquée, est sans doute un phénomène fréquent, au point que nous sommes tous probablement porteurs de nombreuses cellules cancéreuses isolées, alors que c'est seulement l'un d'entre nous parmi trois ou quatre $(28 \%)$ qui développera un cancer au cours de sa vie.

L'expérience montre ainsi que le processus de cancérisation comporte deux étapes successives distinctes: la transformation maligne d'une cellule, puis le développement clonal de cette cellule en une tumeur maligne. Cette deuxième étape met en jeu des phénomènes complexes qui suppriment le caractère stochastique de l'ensemble.

Dans le cas des radiations, ce qu'il y a de remarquable, c'est que la radiation, non seulement provoque la première étape, mais également favorise la seconde par des altérations complexes qui peuvent revêtir des modes très différents (mise en action d'un virus latent préexistant, translocation d'un fragment de chromosome, désordre immunologique, aplasie de certaines fonctions cellulaires ou de certains circuits nutritionnels, etc.) Tout ceci contribue à introduire un seuil. Une seule irradiation massive peut suffire ; c'est ce qui s'est passé à Hiroshima et à Nagasaki dont, selon BEEBE, les résultats n'ont pas finalement permis de trancher le problème du seuil. Le plus souvent, il faut des irradiations répétées, c'est le cas de la leucémie lymphoïde de la souris, de la leucémie myéloïde 
chez l'Homme, des cancers de la peau provoqués par les rayons UV. Le processus progressif de la cancérisation est bien connu des cliniciens. Les lésions précancéreuses encore réversibles sont un signal d'alarme souvent mis à profit. C'est ainsi que si l'on considère non plus la transformation d'une cellule, mais la production de la tumeur maligne, la relation simple de la proportionnalité entre la dose et l'effet disparaît, pour laisser la place à une relation complexe qui implique l'existence non seulement d'un seuil, mais d'un seuil élevé à partir duquel la fréquence augmente avec la dose plus vite que ne le voudrait la proportionnalité. Quand on veut produire des leucémies lymphoïdes chez la souris avec des rayons $X$, il faut toujours dépasser au moins 100 rads.

Le cancérologue ne peut accepter le raisonnement stochastique. Ce raisonnement dirait par exemple: "Supposons que cent mille bouffées de cigarettes produisent un cancer sur deux fumeurs, alors une seule bouffée de cigarette donnée à deux cent mille individus une fois dans leur vie donnera un cancer". Imaginer qu'une seule bouffée de cigarette au cours de la vie puisse produire un cancer relève de Pabstraction, car nous serions tous cancéreux pour avoir inhalé, par la fumée ambiante, bien plus qu'une bouffée.

II ne faut d'ailleurs pas négliger la notion de seuil pratique. En voici un cas (il y en a d'autres) : il est bien établi que la latence d'un radiocancer augmente lorsque la dose diminue. On aboutit à un seuil pratique dès que la latence dépasse la longévité restante. A cet égard, BEEBE a rapporté que, toutes choses égales d'ailleurs, la latence des tumeurs solides induites à Hiroshima et Nagasaki s'est avérée d'autant plus longue que le sujet était plus jeune au moment de l'irradiation, en sorte que, près de quarante ans après, on voit encore apparaître de nouveaux cancers solides radio-induits (la latence des leucémies fut, comme on sait, beaucoup plus courte).

Le Groupe a longuement discuté de cette question. II a admis l'exac titude des considérations précédentes. Mais les représentants de la CIPR ont fait remarquer qu'elles ne fournissent, dans notre ignorance actuelle, aucune donnée quantitative de prévision. C'est pourquoi, on s'est mis d'accord pour admettre le raisonnement stochastique qui prévaut actuellement, avec ses conséquences, tout en reconnaissant :

$1^{\circ}$ qu'il est inexact, et qu'on ne le conserve que pour les besoins de la cause ;

$2^{\circ}$ qu'il conduit à une surestimation du risque.

\section{LA RADIATION NATURELLE ET LE POINT ZÉRO}

On ne peut pas oublier que toute radiation artificielle s'ajoute à la radiation naturelle, et l'on doit prendre celle-ci en considération dès P'instant qu'on a affaire à des débits et à des doses du même ordre. Cette notion vient au premier plan lorsqu'il s'agit de fixer le point zéro, c'est-à-dire la dose à partir de laquelle tout incrément artificiel peut être vu comme le responsable potentiel d'un détriment. Certains prétendent que ce point zéro se trouve au niveau de l'irradiation naturelle, quelle qu'elle soit, c'està-dire où qu'on soit, en sorte qu'aucun apport artificiel ne doit être négligé. Cette position n'est pas réaliste. 
En effet, l'irradiation naturelle varie approximativement entre 100 et 300 mrem par an selon l'altitude et la nature du sol (à l'exception de quelques "points chauds" peu étendus). Le Groupe a été unanime pour admettre qu'aucune statistique n'a révélé une augmentation du détriment dans les zones à 300 mrem, par rapport aux zones alluviales à 100 mrem. Certes, les statistiques qui concernent les populations des hauts plateaux andins sont peu précises (du fait du faible développement du secteur médico-social). En revanche, d'excellentes statistiques ont été faites dans des territoires à haute radioactivité naturelle, notamment en Scandinavie, aux USA, en Grande-Bretagne et en France. Enfin, de très grandes villes comme Denver et Mexico ont fourni des statistiques crédibles. Aucune augmentation significative du détriment radiobiologique n'est apparue.

Certains, à ce sujet, ont posé une question qui reste conjecturale : la vie s'étant développée sous cette irradiation naturelle, n'est-il pas permis de penser que l'évolution a sélectionné des races qui lui sont parfaitement adaptées, au point de tirer profit de cette irradiation. Certains vont même plus loin, et prétendent que les faibles doses administrées à faible débit, comme l'irradiation naturelle, produisent des effets bénéfiques (jusqu'à 300 mrad par an).

Le cas du Kerala n'est pas résolu. Dans cette région de l'Inde une population importante reçoit une irradiation naturelle nettement plus abondante (de l'ordre de 1 rem par an). Les vivants y ont actuellement accumulé une dose estimée à 2 millions d'hommes-rems, bien que la longévité moyenne n'y soit que de 45 ans (pour des raisons indépendantes des radiations). Une statistique convenable serait du plus haut intérêt, quelle qu'en soit la réponse. La chose est difficile et onéreuse. II n'est pas certain que l'échantillon de population soit suffisant pour donner une modification significative de l'incidence des cancers. En Chine, un rapport négatif vient d'être publié sur les résultats enregistrés dans des circonstances comparables. Une commission mixte Inde - AIEA - NCI (1) vient d'étudier sérieusement les conditions dans lesquelles devrait être conduite une étude statistique pour donner des résultats (positifs ou négatifs) significatifs. Etant donné la dimension de la population, sa dispersion, son hétérogénéité et sa mobilité, l'étude devrait être poursuivie pendant vingt années consécutives. Le budget approximatif de P'opération a été établi. II est suffisamment élevé pour avoir découragé jusqu'ici les Indiens d'engager l'affaire. Quant aux Américains, ils n'éprouvent pas sur ce problème une motivation comparable à celle d'Hiroshima. L'AIEA en aura-telle les moyens?

De tout ceci il résulte qu'on peut en toute sécurité mettre le point zéro à 300 mrem (sans exclure la possibilité de le remonter ultérieurement). Ceci signifie qu'en un lieu où l'irradiation naturelle est de $100 \mathrm{mrem}$, une irradiation artificielle supplémentaire de 200 mrem reste en-dessous du point zéro (2).

(1) $\mathrm{NCl}$ : National cancer institute, USA.

(2) J'ai entendu récemment soutenir au sein d'une Commission nationale que la radioactivité à la surface du sol au-dessus d'un entrepôt de déchets nucléaires ne devrait pas dépasser 100 millirems par an. Dans certaines régions ce serait alors l'endroit le plus favorable pour construire sa maison. 
Ceci signifie encore qu'à ceux qui prétendent que toute protection supplémentaire est bénéfique, et qu'il n'y a pas de niveau où la protection doive s'arrêter, on peut répondre : "Si, il y en a un, c'est celui des fluctuations de l'irradiation naturelle". Notons que la référence quantitative à l'irradiation naturelle est la plus "parlante" auprès du grand public. Celui-ci ne peut décider si 50 mrem par an constituent un risque, mais il comprend qu'il est puéril d'exiger, autour de Fessenhein, un niveau de radiation inférieur à celui qui règne naturellement dans les proches vallées vosgiennes, vallées que personne ne songe à évacuer. C'est en comparant les pollutions autour de Three Mile Island à des durées de séjour à Mexico ou à Val d'Isère qu'on s'est fait le mieux entendre.

\section{LES CONCEPTS VAGUES}

Au cours de cette réunion, ainsi qu'il advient le plus souvent lorsqu'une commission traite du problème des doses admissibles, certaines discussions oiseuses se sont déroulées par la mise en avant de concepts vagues (pour ne pas dire fumeux) qui conduisent à des dialectiques sans issue, donc sans fin, précisément parce qu'ils sont vagues. On doit reconnaître que certains scientistes préfèrent la discussion à la vérité, et vont même jusqu'à redouter celle-ci car elle met un terme à la discussion.

L'un des concepts qu'on avance fréquemment en radioprotection est celui de la valeur d'une vie humaine. Pour évaluer la valeur d'une grandeur il faut une unité. Quelle peut-elle être ici ? En fait il n'y en a pas. Ce n'est ni un coût ni un prix. Une vie humaine n'a pas de prix, la septième symphonie de Beethoven non plus, pour cette raison qu'on ne peut créer ni l'une ni l'autre avec de l'argent. Si tel amateur ou tel musée acquiert une toile de Rembrandt pour $\mathrm{n}$ dollars, cette somme ne représente pas le prix de la toile (puisqu'elle ne pourra la recréer si la toile est détruite), mais seulement le prix du plaisir qu'on éprouve à la posséder ou des avantages qu'on peut en tirer.

On nous indique, parfois, ce que "coûte un soldat" au cours d'une guerre. Mais ce n'est pas le coût de sa vie, c'est celui de sa mort. Ne cherchez donc pas à évaluer jusqu'où l'on peut aller financièrement pour "sauver une vie humaine", expression qui, quantitativement ne veut rien dire. En effet, comment comparer quantitativement la vie de Durand à 15 ans à sa vie à 65 ans ? et la vie de Durand à 30 ans à celle de Mozart au même âge ? Non, de grâce, ne vous engagez pas dans ce lacis; vous n'en sortirez pas. Faut-il avouer qu'on n'a pas réussi à donner une définition précise au mot "optimisation" qui pourtant figure dans le titre de cette réunion ? Certains veulent qu'optimiser c'est diminuer les doses autant qu'il est possible audessous des limites permises. Cette définition dangereuse implique que les doses permises ne garantissent pas une réelle sécurité ; sinon pourquoi cherchez mieux? C'est pourquoi j'ai proposé une définition plus large (" procédures pour obtenir mieux") sans référer aux doses admissibles, laissant entendre comme on a vu au paragraphe 3 , que la zone de fluctuation des doses naturelles est une zone sûre, que chercher plus loin devient superflu, que l'optimisation s'arrête là. Mais on ne s'est pas mis d'accord...

On pourrait en dire autant du terme de "justification" si cher à la CIPR, dont on a beaucoup parlé sans parvenir à le définir, etc. 


\section{LA COMPARAISON AVEC LES POLLUANTS CHIMIQUES GÉNOTOXIQUES}

Le problème de l'optimisation n'est pas limité aux radiations ionisantes. II concerne tout autant les composés chimiques génotoxiques (mutagènes et cancérigènes). Ces risques s'additionnent fréquemment. Se protéger contre un seul ne suffit pas. Cette considération est vite apparue lorsqu'on a discuté de la question : quelle somme faut-il dépenser pour supprimer un homme-rem ? II est clair que les ressources dont dispose l'optimisation ne sont pas illimitées, et qu'il convient de faire pour le mieux avec ce dont on dispose. II est clair également que la protection contre les radiations a atteint un très haut niveau, très au-dessus de celle qui existe le plus souvent contre les génotoxiques chimiques. Un exemple très édifiant est celui de l'oxyde d'éthylène dans les unités de stérilisation froide où, par des mesures simples et peu coûteuses, on pourrait améliorer considérablement la situation actuelle.

Disons que s'il faut $\mathrm{n}$ dollars pour supprimer un homme-rem (LINDELL pense de 100 à 150 dollars) on supprimerait de nombreux hommes-rems équivalents d'oxyde d'éthylène avec ces $n$ dollars. Dans ces conditions, utiliser $n$ dollars à protéger contre les radiations équivaut, dans de nombreux cas, à aider les riches à s'enrichir sans se préoccuper de ce qu'il advient aux pauvres. En sorte que si l'homme politique de décision me disait "Je dispose de $x$ dollars pour améliorer la protection contre la pollution génotoxique ; combien dois-je en réserver pour améliorer la situation autour des réacteurs nucléaires ?" (et c'est bien cela l'optimisation), je lui répondrais: "Dans la situation actuelle aucun. Vous avez bien mieux à faire ailleurs". Reconnaissons à ce sujet les remarquables assainissements de Patmosphère de certaines grandes villes comme Londres et Pittsburgh depuis vingt-cinq ans. La contamination de New York par le soufre a diminué de $95 \%$ au cours des dix dernières années.

\section{LA CIPR}

Depuis sa création en 1928, et surtout depuis 1950, la Commission internationale de protection radiologique a joué un rôle important et utile. Elle a réussi à édicter des règles de sécurité qui ont aujourd'hui force de loi dans la quasi-totalité des pays. En quel autre domaine des risques peuton se flatter d'un pareil accomplissement?

Grâce à la CIPR la pratique radiologique ne produit plus d'accident, ni chez les malades, ni chez les médecins qui, jusqu'à la deuxième guerre mondiale, payèrent un lourd tribut à l'exercice de leur art. Grâce à la CIPR les centrales nucléaires sont construites et opèrent selon des normes qui garantissent une exceptionnelle sécurité dans un domaine a priori plein de dangers.

Ce que le public attend avant tout est une réponse claire à la question : "Suis-je en sécurité"? La CIPR a répondu avec précision :"Vous êtes en sécurité lorsque telle et telle condition sont satisfaites". 
Mais une fois cette tâche magnifiquement accomplie, que faire si l'on continue d'exister? Qu'on pardonne ma franchise. J'ai le sentiment qu'on complique les choses, qu'on coupe les cheveux en quatre, qu'on gonfle des périls mineurs, voire imaginaires, qu'on complique des procédures, et qu'on les ouvre à des discussions sans issue. On revient sur la réponse claire que l'on avait donnée, et l'on avance des nuances qui, pour le public jusqu'alors rassuré, signifient qu'après tout on n'est jamais en sécurité, d'où de nouvelles inquiétudes. Oublie-t-on donc, que sur cette terre la sécurité n'est jamais qu'un risque accepté ? II est dès lors indispensable de dissocier les problèmes au niveau de la connaissance scientifique des mêmes problèmes au niveau pratique. C'est ce que la CIPR a justement fait avec ses recommandations. Pouquoi y revenir ?

$\mathrm{Si}$ je devais donner un conseil à cette institution que, je le répète, j’admire, je lui dirais d'ouvrir son champ d'action à celui de l'industrie chimique génotoxique, champ beaucoup plus vaste et divers que celui des radiations, et à la mise en ordre duquel ce qu'elle a acquis et réalisé dans le champ des radiations lui servirait de modèle et de guide. 